\title{
Differential Expression of CD24 and its Significance in Normal Endometrium, Hyperplastic Lesions and Endometrial Carcinoma
}

\author{
REHAB K. MOHAMMED YOUSEF, M.D. \\ The Department of Pathology, Faculty of Medicine, Minia University, Egypt
}

\begin{abstract}
Background: Endometrial carcinoma is considered the sixth most common cancer in women worldwide comprising $4 \%$ of all cancers in women. CD24 protein was originally reported to be expressed by pre-B lymphocytes. This protein had been recognized only as a cell marker for hematopoietic cell lineages. CD24 expression is considered as an important biomarker for diagnosis, disease progression and cancerrelated death in many solid tumors, like breast, colonic and ovarian carcinomas.
\end{abstract}

Aim: The present work was conducted to study the immunohistochemical expression of CD24 in normal cyclic endometrium, hyperplastic endometrium and endometrial carcinoma, in order to demonstrate its expression pattern and to examine its association with various clinicopathological variables in endometrial carcinoma cases.

Material and Methods: The present study comprised 110 cases of endometrial tissue: 20 cases $(18.2 \%)$ of cyclic endometrium, 30 cases $(27.3 \%)$ of endometrial hyperplasia without atypia, 25 cases $(22.7 \%)$ of atypical endometrial hyperplasia and 35 cases $(31.8 \%)$ of endometrioid carcinoma. Immunohistochemistry was performed using the avidin biotinperoxidase complex method.

Results: CD24 immunostaining was membranous and cytoplasmic. In the normal cyclic endometrium, membranous CD24 showed down-regulation in the proliferative phase and up-regulation in the secretory phase. Both membranous and cytoplasmic CD24 expression was statistically increase in endometrial carcinoma and reduced in hyperplastic lesion $p<0.001$ and $p=0.004$ respectivebly. Among cases of carcinoma, membrnuos CD24 expression was statistically significant with grade, stage and myometrial invasion $(p=0.040 \& p=0.044$ and $p=0.036$ respectively). Cytoplasmic expression showed significant association with grade only $(p=0.037)$. Membranous CD24 had $71.4 \%$ sensitivity and $76 \%$ specificity for differentiation of endometrioid carcinoma from atypical EH. Cytoplasmic CD24 had low sensitivity (40\%) but high specificity (80\%) for distinction of endometrioid carcinoma from atypical endometrial hyperplasia. Total CD24 had 77.2\% sensitivity and $76 \%$ specificity for distinction of atypical endometrial hyperplasia from endometrioid carcinoma.

Correspondence to: Dr. Rehab K. Mohammed Yousef, E-Mail: rehab kemo@yahoo.com
Conclusion: Membernous CD24 was expressed in a cyclic pattern in the normal endometrium. CD24 expression was increased in case of endometrial carcinoma than hyperplastic lesion. These results suggest that CD24 can be a useful as diagnostic marker in differentiation between endometrial hyperplasia and carcinoma. Also CD24 implicated in progression of endometrial carcinoma. Total CD24 expression (membernous and cytoplasmic) is more specific and more sensitive in differentiation of atypical endometrial hyperplasia from endometrioid carcinoma.

Key Words: CD24 - Normal cyclic endometrium - Endometrimal hyperplasia - Endometroid carcinoma Immunohistochemistry

\section{Introduction}

WORLDWIDE endometrial carcinoma is considered the sixth most common cancer in women, comprising $4 \%$ of all cancers in women [1]. In developed countries it is the most common gynecologic malignancy and the second most common tumor in developing countries; also it is the seventh leading cause of death among women [2]. In Egypt, it represents $14.72 \%$ of female genital tract malignancies [3] and $0.62 \%$ of all female cancers [4] .

CD24 protein was originally reported to be expressed at early stages of-B lymphocytes maturation. It is had been recognized only as a cell marker for hematopoietic cell lineages [5]. It is called the heat-stable protein due to its heat resistance [6]. CD24 is expressed on hematopoietic cells, including $\mathrm{T}$ and $\mathrm{B}$ cells $[\mathbf{7 , 8 ]}$; neutrophils and eosinophils $[9,10]$, dendritic cells and macrophages [7,11]. In many solid tumors, like breast, colonic and ovarian carcinomas, CD24 expression is considered as an important marker for diagnosis, disease progression and cancer-related death [12-17].

Few reports about CD24 expression in normal cyclic endometrium and other endometrial lesions were conducted, so this study is done to demon- 
strate the role of CD24 in normal cyclic endometrium, hyperplastic lesion and endometrial carcinoma of Egyptian females.

\section{Material and Methods}

\section{Patients' and samples characteristics:}

This study was conducted at Pathology Department, Minia University from Augest 2017 to May 2018 .

This study included 110 cases of endometrial tissue: 20 cases $(18.2 \%)$ of cyclic endometrium (10 cases were proliferative and 10 were secretory endometrium), 30 of endometrial hyperplasia cases $(27.3 \%)$ of $(\mathrm{EH})$ without atypia, 25 cases $(22.7 \%)$ of atypical EH and 35 cases (31.8\%) of Endometrioid Carcinoma (EC) diagnosed in Pathology Department, Minia University Hospital and Minia Oncology Center, Egypt during the period from April 2009 to March 2017.

Hematoxylin and eosin stained slides were reviewed to confirm the diagnoses of the cases. Cases were diagnosed according to WHO based classification [18], grouped into EH without atypia, $\mathrm{EH}$ with atypia, and endometrioid carcinoma. Grading of endometrioid carcinoma was done according to the FIGO criteria [18] . Staging of endometrioid carcinoma was done according to the FIGO 2009 staging criteria [19].

\section{Results}

The patient mean age \pm SD, median age and age range for each group included in this study was $37.2 \pm 4.6$ years, 38 years and $33-42$ for cyclic endometrium, $61.9 \pm 6.9$ years, 62 years and $49-72$ in EH without atypia, $60 \pm 10.5$ years, 61 years and 39-84 for atypical EH and $64.9 \pm 6.7$ years, 64 years and 49-80 for endometrioid EC.

Among EC group, the median age was 64 years old, $15 / 35(42.9 \%)$ were $<64$ and 20/35 (57.1\%) were $>64$. Regarding tumor grade, $15 / 35(42.8 \%)$ cases were grade $1,10 / 35(28.6 \%)$ cases were grade 2 and $10 / 35(28.6 \%)$ cases were grade 3 . Myometrial invasion $<1 / 2$ was present in 22/35 $(62.9 \%)$ of cases, while $13 / 35(37.1 \%)$ showed myometrial invasion $\geq 1 / 2$. Seventeen cases out of twenty two $17 / 22(77.2 \%)$ were negative for $\mathrm{LN}$ metastasis and 5/22 (22.8\%) were positive for LN metastasis.

Regarding FIGO stage of the tumor; 29/35 $(82.9 \%)$ cases were stage I, $2 / 35(5.7 \%)$ cases were stage II, $3 / 35(8.5 \%)$ cases were stage III and $1 / 35$ $(2.8 \%)$ case was stage VI. These cases were cate- gorized into two groups, one group included cases of stage I [29/35 (82.9\%)] and the other group included stage II/III/IV cases [6/35 (17.1\%)]. This was done for statistical purpose.

\section{Immunohistochemistry method:}

Tissue sections were prepared then deparaffnized and rehydrated through xylene and graded ethanol solutions and then slides incubated for 5 min with $3 \%$ hydrogen peroxide to block the endogenous peroxidase activity. Antigen retrieval was done according to manufacturer's instructions. Then slides incubated with the primary antibodies overnight in moist chamber. The used primary antibody for $\mathrm{CD} 24$ is ( 0.1 concentrated monoclonal mouse antibody at 1:100 concentration (clone SN3b Biocare Medical) then slides washed in PBS buffer. The avidin-biotin detection kit using diaminobenzidine (DAB) as chromogen were used to detect antibody reaction. Slides were counterstained with Mayers hematoxylin for 15 seconds, then dehydrated through graded alcohol solutions, cleared in xylene and coverslipped. Negative control was done by omitting the primary antibody. Ovarian serous carcinoma was used as positive control and tested on each run.

\section{Immunohistochemical analysis:}

CD24 antibody, positive staining was detected when a membranous and/or cytoplasmic staining was observed. CD24 expression was grouped into positive and negative cases according to [20]. Negative cases had to show definitely no immunoreactivity in any part of the section. All of the other cases, beginning with a weak but unequivocal staining of the cells, were defined as positive.

\section{Statistical analysis:}

Mean, standard deviation, frequency distribution and cross tabulation have been performed. Chisquare and fixer exact test were used to compare categorical variables. Results were considered statistically significant when $p$-value $<0.05$. Data were analyzed using the Statistical Package for Social Sciences (SPSS) Version 18 software. To determine the diagnostic efficacy of CD24, the numbers of True-Positive (TP), True-Negative (TN), False-Positive (FP), and False-Negative (FN) cases were determined for the marker. Accordingly, the sensitivity, specificity, Positive Predictive Values (PPV), Negative Predictive Values (NPV) and diagnostic accuracy of CD24 were calculated using MedCalc statistical software.

\section{Immunoreactivity for CD24:}

CD24 immunostaining was membranous and cytoplasmic; each subcellular localization was 
separately evaluated and statistically analyzed. Then combined membranous and cytoplasmic CD24 immunostaining was evaluated.

\section{1- Membranous CD24 expression in different his- topathological groups.}

As regard cyclic endometrium, 9/20 (45\%) cases were positive. Among them, 2/10 cases of proliferative phase (20\%) were positive. Converse-

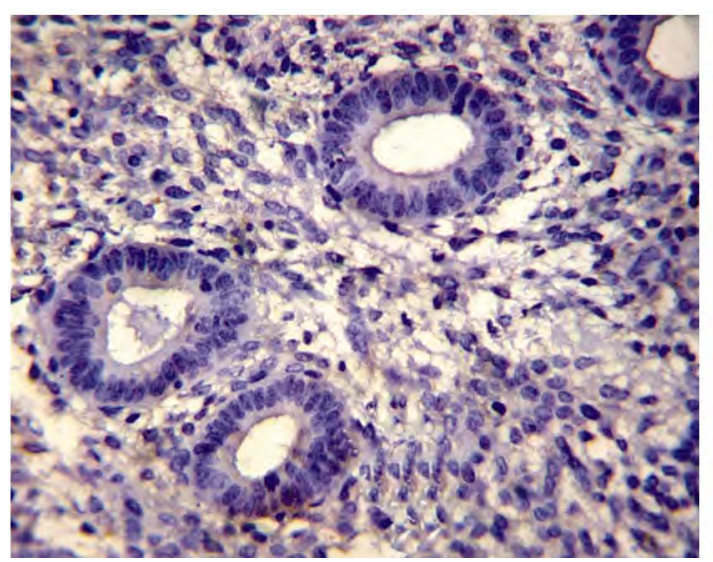

Fig. (1A): Proliferative endometrium showing negative CD24 expression (DAB chromogen, haematoxylin counter stain X400).

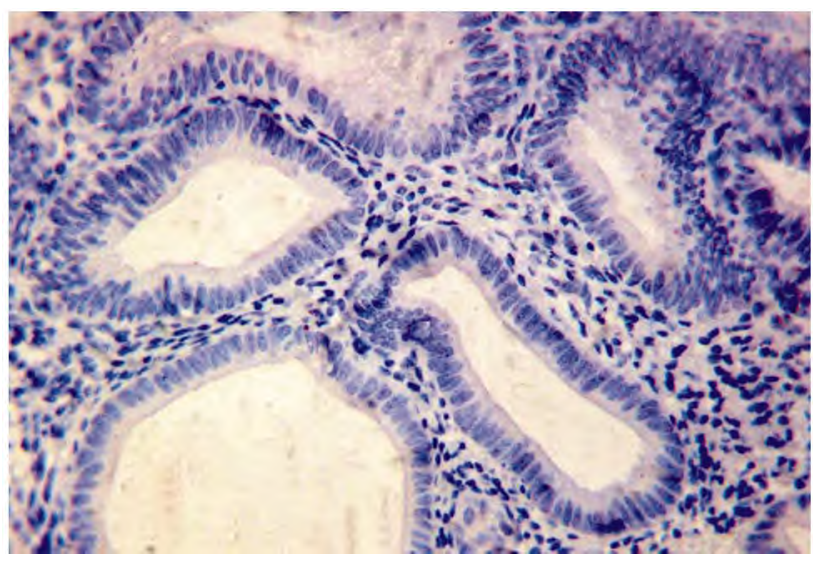

Fig. (2A): Endometrial hyperplasia without atypia showing negative CD24 expression (DAB chromogen, haematoxylin counter stain X400).

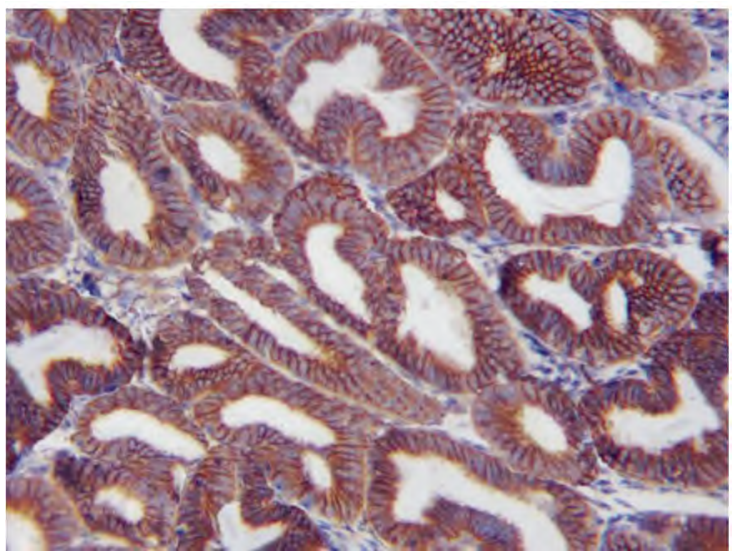

ly, $7 / 10$ cases $(70 \%)$ of secretory phase cases were positive and $3 / 10$ cases $(30 \%)$ were negative Fig. (1 A,B). In EH without atypia and atypical EH cases, majority of cases 26/30 (86.6\%), 19/25 (76\%) respectively) were negative for the membranous CD24 immunoreactivity Fig. (2A,B). Positive membranous CD24 expression in endometrioid carcinoma cases was detected in 25/35 (71.4\%) cases Fig. (3).

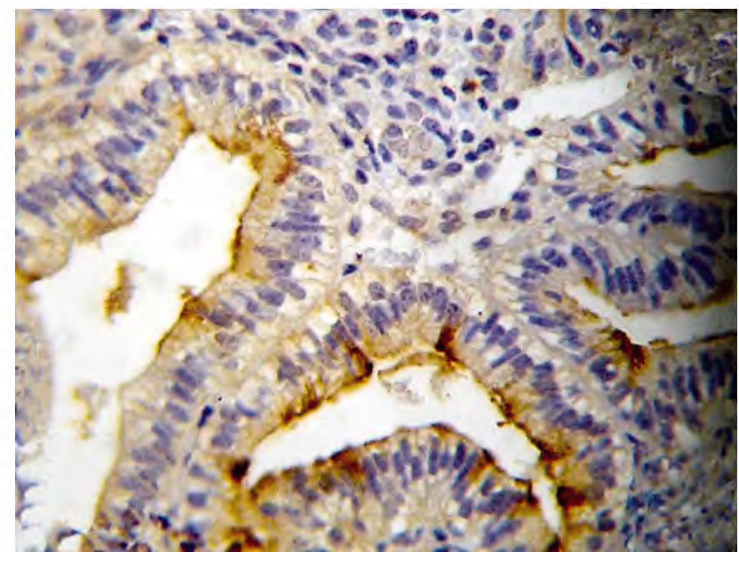

Fig. (1B): Secretory endometrium showing positive CD24 expression (DAB chromogen, haematoxylin counter stain X400).

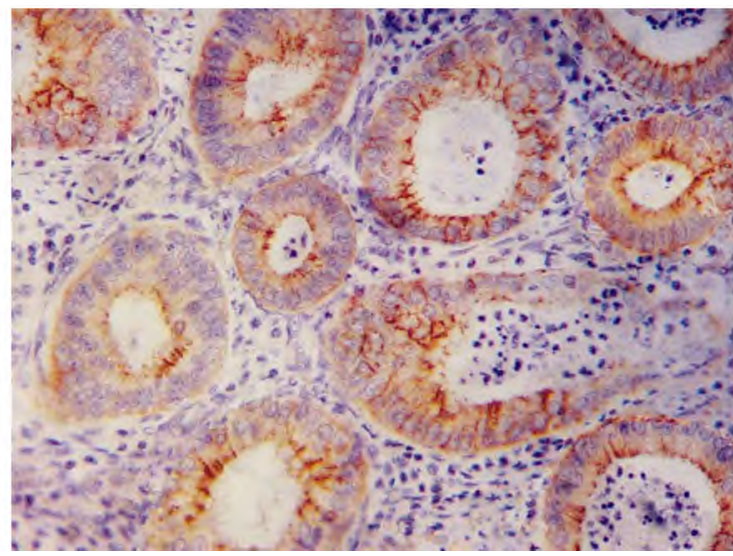

Fig. (2B): Atypical endometrial hyperplasia showing positive membranous CD24 expression (DAB chromogen, haematoxylin counter stain X400).

Fig. (3): Well differentiated endometrioid carcinoma showing positive membranous CD24 expression (DAB chromogen, haematoxylin counter stain X200). 
Association between membranous CD24 expression and different histopathological groups:

Among the histopathological groups a statistically significant association was found in the membranous CD24 expression $(p<0.001)$. There was statisically significant increase in the expression of membranous CD24 in secretory phase cases as compared in proliferative phase cases $(p=0.02)$.

A statistically significant association in membranous CD24 immunostainining in cyclic endometrium cases as compared to its expression in EH without atypia, atypical EH cases and endometrioid carcinoma cases were found ( $p=0.04, p=0.012$ and $p=0.042$, respectively).

As regards cases of $\mathrm{EH}$ no statistically significant difference was found between expression among cases of $\mathrm{EH}$ without atypia and atypical $\mathrm{EH}$ cases $(p=0.307)$.

A statistically significant difference in the immunoexpression of membranous CD24 in endometrioid carcinoma cases as compared to its expression in $\mathrm{EH}$ without atypia and atypical $\mathrm{EH}$ cases was detected ( $p<0.001$ for both).

For membranous CD24 immunolocalization a statistically significant difference between atypical EH compared with well-differentiated endometrial carcinoma was found $(p=0.023)$.
The diagnostic validity of membranous CD24 immunoreactivity:

Sensitivity, specificity, PPV, NPV and accuracy of membranous CD24 in differentiating endometrioid carcinoma from atypical EH were shown in (Table 1). Membranous CD24 had 71.4\% sensitivity and $76 \%$ specificity for differentiation of endometrioid carcinoma from atypical $\mathrm{EH}$.

The diagnostic validity of membranous CD24 in distinction of well differentiated endometrioid carcinoma from atypical $\mathrm{EH}$, had much lower sensitivity $(46.7 \%)$.

Table (1): The diagnostic validity of membranous CD24 immunoreactivity.

\begin{tabular}{lccccc}
\hline Diagnosis & Sensitivity & Specificity & PPV NPV & Accuracy \\
\hline - Atypical EH Vs. & $71.4 \%$ & $76 \%$ & $80.6 \%$ & $65.5 \%$ & $73.3 \%$ \\
$\begin{array}{l}\text { carcinoma } \\
\text { Atypical EH Vs. }\end{array}$ & $46.7 \%$ & $76 \%$ & $61.5 \%$ & $65.5 \%$ & $45 \%$ \\
$\begin{array}{l}\text { well } \\
\text { differentiated } \\
\text { carcinoma }\end{array}$ & & & & & \\
\hline
\end{tabular}

PPV: Positive Predictive Value. NPV: Negative Predictive Value.

Association between membranous CD24 expression and clinicopathological variables ofendometrioid carcinoma cases (Table 2):

There was statistically positive significant association between membranous CD24 immunostaining and tumor grades $(p=0.040)$. All cases of grade 3 were positive compared to $(7 / 10) 70 \%$ and $(8 / 15) 53.3 \%$ for grade 2 and grade 1 , respectively.

Table (2): Association between membranous and cytoplasmic CD24 immunostaining and clinicopathological variables of endometrioid carcinoma cases.

\begin{tabular}{|c|c|c|c|c|c|c|c|}
\hline \multirow{2}{*}{$\begin{array}{l}\text { Clinicopathological } \\
\text { variables }\end{array}$} & \multirow{2}{*}{ No. } & \multicolumn{2}{|c|}{ Membranous expression } & \multirow{2}{*}{$\begin{array}{c}p- \\
\text { value }\end{array}$} & \multicolumn{2}{|c|}{ Cytoplasmic expression } & \multirow{2}{*}{$\begin{array}{c}p- \\
\text { value }\end{array}$} \\
\hline & & $\begin{array}{l}\text { Negative } \\
(\%)\end{array}$ & $\begin{array}{l}\text { Positive } \\
(\%)\end{array}$ & & Negative & Positive & \\
\hline \multicolumn{8}{|l|}{ Age: } \\
\hline$<64$ & 15 & $3(20)$ & $12(80)$ & 0.091 & $7 \quad(46.7)$ & $8(53.3)$ & 0.156 \\
\hline$>64$ & 20 & $8(40)$ & $12(60)$ & & $14(70)$ & $6(30)$ & \\
\hline \multicolumn{8}{|l|}{ Grade: } \\
\hline Grade 1 & 15 & $7(46.7)$ & $8 \quad(53.3)$ & 0.040 & $13(86.7)$ & $2(13.3)$ & 0.037 \\
\hline Grade 2 & 10 & $3(30)$ & $7 \quad(70)$ & & $5 \quad(50)$ & $5(50)$ & \\
\hline Grade 3 & 10 & 0 & $10(100)$ & & $4 \quad(40)$ & $6(60)$ & \\
\hline \multicolumn{8}{|l|}{ Myometrial invasion: } \\
\hline$<1 / 2$ & 22 & $9(40.9)$ & $13(59.1)$ & 0.036 & $16(72.7)$ & $6(27.3)$ & 0.116 \\
\hline$>1 / 2$ & 13 & $1(7.7)$ & $12(92.3)$ & & $6 \quad(46.2)$ & $7(53.8)$ & \\
\hline \multicolumn{8}{|l|}{ LN metastasis: } \\
\hline Negative & 17 & $5(29.4)$ & $12(70.6)$ & 0.168 & $11(64.7)$ & $6(35.3)$ & 0.323 \\
\hline Positive & 5 & 0 & $5 \quad(100)$ & & $2(40)$ & $3(60)$ & \\
\hline \multicolumn{8}{|l|}{ FIGO stage: } \\
\hline Stage I & 29 & $10(34.5)$ & $19(65.5)$ & 0.044 & $18(62.1)$ & $11(37.9)$ & 0.447 \\
\hline Stage II-III-IV & 6 & 0 & $6 \quad(100)$ & & $3 \quad(50)$ & $3 \quad(50)$ & \\
\hline
\end{tabular}

Test of significance: Chi-square test and fisher exact test, Significant $p$-value $<0.05$. 
As regards myometrial invasion a statistically significant positive association between membranous CD24 immunostaining and myometrial invasion $(p=0.036)$, the positivity was higher among cases had $>1 / 2$ myometrial invasion in which $12 / 13$ cases $(92.3 \%)$ that had $>1 / 2$ myometrial invasion were positive to membranous CD24 immunostaining. Statistically significant association was found between membranous CD24 immunostaining and FIGO stage of carcinoma $(p=0.044)$. All advanced stage cases 6/6 (100\%) showed positive expression compared to early stage cases that had positivity in $19 / 29(65.5 \%)$ of cases.

No statistically significant association was found between membranous CD24 immunoexpression and patients' age and LN status ( $p=0.091 \&$ $p=0.168$ respectively).

\section{2- Cytoplasmic CD24 expression in different his-} topathological groups:

The present study demonstrated statistically significant difference between the expression of cytoplasmic CD24 among the different histopathological groups $(p=0.022)$. No statistically significant difference was found among normal cyclic endometrium $(p=0.276)$.

There was a statistically significance in the expression of cytoplasmic CD24 in cyclic endometrium cases as compared to its expression in $\mathrm{EH}$ without atypia cases $(p=0.011)$. However no significant differences were detected between cases of cyclic endometrium and atypical EH cases or endometrioid carcinoma cases $(p=0.293$ and $p=$ 0.847 , respectively).

As comparing the immunocytoplasmic expression of CD24 between cases of EH without atypia and endometrioid carcinoma cases a statistically significant increase in the expression was detected $(p=0.004)$.

Between cases of atypical EH cases and EH without atypia cases or endometrioid carcinoma cases no statistically significant differences were detected ( $p=0.140$ and $p=0.153$, respectively). Also there was no statistically significant difference was found between cytoplasmic CD24 expression in atypical $\mathrm{EH}$ compared with well-differentiated endometrial carcinoma Fig. $(4 \mathrm{~A}, \mathrm{~B})(p=1.00)$.

The diagnostic validity of cytoplasmic CD24 immunoreactivity:

Sensitivity, specificity, PPV, NPV and accuracy of cytoplasmic CD24 in differentiating endometrioid carcinoma from atypical EH were shown in (Table 3).
Cytoplasmic CD24 had low sensitivity (40\%) but high specificity $(80 \%)$ for distinction of endometrioid carcinoma from atypical $\mathrm{EH}$.

As regard CD24 positive cytoplasmic immunoexpression in diagnosis of well differentiated endometrioid carcinoma in comparison with atypical EH, cytoplasmic CD24 had much lower sensitivity $(13.3 \%)$.

Table (3): The diagnostic validity of cytoplasmic CD24 immunoreactivity.

\begin{tabular}{lccccc}
\hline Diagnosis & \multicolumn{2}{c}{ Sensitivity Specificity } & PPV & NPV & Accuracy \\
\hline - Atypical EH Vs. & $40 \%$ & $80 \%$ & $73.6 \%$ & $48.7 \%$ & $56.6 \%$ \\
$\begin{array}{l}\text { carcinoma } \\
\text { Atypical EH Vs. } \\
\text { well }\end{array}$ & $13.3 \%$ & $80 \%$ & $28.5 \%$ & $60.6 \%$ & $36.6 \%$ \\
$\begin{array}{l}\text { differentiated } \\
\text { carcinoma }\end{array}$ & & & & & \\
\hline
\end{tabular}

PPV : Positive Predictive Value.

NPV : Negative Predictive Value.

Association between cytoplasmic CD24 expression and clinicopathological variables of endometrioid carcinoma:

The association of cytoplasmic CD24 expression with clinicopathological variables of endometrioid carcinoma cases was summarized in (Table 2).

This study demonstrate statistically significant positive association between cytoplasmic CD24 immunostaining and tumor grades $(p=0.037)$. The frequency rate of positive cytoplasmic CD24 expression was highest in grade $3[6 / 10(60 \%)$ compared to $2 / 15(13.3 \%)$ and $5 / 10(50 \%)$ for grade 1 and 2 , respectively].

Regarding other clinicopathological variables and cytoplasmic CD24 expression no statistically significant associations were detected.

3- Combinedmembranous and cytoplasmic CD24 expression in histopathological groups:

In cyclic endometrium, 9/20 (45\%) were totally negative for CD24, $5 / 20$ cases $(25 \%)$ were positive for both expressions and $6 / 20$ cases $(30 \%)$ were -veMEM/+veCYTO or +veMEM/-veCYTO.

In EH without atypia, most cases 26/30 (86.6\%) were totally negative for $\mathrm{CD} 24$, while $2 / 30$ cases (6.7\%) were positive for both expressions and 2/30 cases $(6.7 \%)$ were +veMEM/-veCYTO.

Among cases of atypical EH, most cases 19/25 (76\%) were totally negative for CD24, and $1 / 25$ (4\%) was +veMEM/-veCYTO while both staining qualities showed a rate of concordance in $5 / 25$ cases $(20 \%)$. 
Regarding endometrioid carcinoma cases, the total CD24 expression was seen in $77.1 \%$ of cases, $16 / 35$ cases $(45.7 \%)$ showed veMEM/+veCYTO or +veMEM/veCYTO patterns of expression and both staining qualities showed a rate of concordance in $31.4 \%$ of cases.

Table (4): Combined membranous and cytoplasmic CD24 expression in different histopathological groups.

\begin{tabular}{|c|c|c|c|c|}
\hline \multirow[b]{2}{*}{ Histopathological groups } & \multirow[b]{2}{*}{ No. } & \multirow{2}{*}{$\begin{array}{c}\text { Negative } \\
\text {-ve/-ve } \\
(\%)\end{array}$} & \multicolumn{2}{|c|}{ Positive } \\
\hline & & & $\begin{array}{l}\text {-ve/+ve or } \\
\text { +ve/-ve }(\%)\end{array}$ & $\begin{array}{c}\text { +ve/+ve } \\
(\%)\end{array}$ \\
\hline Cyclic endometrium & 20 & $9 \quad(45)$ & $6 \quad(30)$ & $5 \quad(25)$ \\
\hline EH without atypia & 30 & $26(86.6)$ & $2(6.7)$ & $2(6.7)$ \\
\hline Atypical EH & 25 & $19(76)$ & 1 (4) & $5 \quad(20)$ \\
\hline Endometrioid carcinoma & 35 & $8 \quad(22.9)$ & $16(45.7)$ & $11(31.4)$ \\
\hline
\end{tabular}

Association between combined membranous and cytoplasmic CD24 immunostaining and histopathological groups:

The present study showed a statistically significant difference between combined membranous and cytoplasmic CD24 immunostaining among the histopathological groups $(p<0.001)$. Endometrioid carcinoma cases demonstrate the highest frequency rate of combined positive membranous and cytoplasmic expression.

A statistically significant increase in combined positive CD24 immunostaining in cyclic endometrium cases as compared to its expression in $\mathrm{EH}$ without atypia cases was found $(p=0.007)$.

However no statistically significant difference was found between cyclic endometrium cases and atypical $\mathrm{EH}$ cases or endometrioid carcinoma cases $(p=0.283$ and $p=0.225$, respectively).

The combined positive CD24 immunostaining in endometrioid carcinoma cases as compared to its expression in $\mathrm{EH}$ without atypia and atypical $\mathrm{EH}$ cases showed statistically significant increase in the expression ( $p<0.001$ and $p=0.002$, respectively). Between cases of $\mathrm{EH}$ without atypia and atypical EH cases no statistically significant difference was detected $(p=0.230)$.

The diagnostic validity of total CD24 immunoreactivity:

Sensitivity, specificity, PPV, NPV and accuracy of total $\mathrm{CD} 24$ in differentiating endometrioid carcinoma from atypical $\mathrm{EH}$ were shown in (Table 5).
Total CD24 had $77.2 \%$ sensitivity and $76 \%$ specificity for distinction of atypical EH from endometrioid carcinoma. Regarding its diagnostic validity in distinction of well differentiated endometrioid carcinoma from atypical EH, total CD24 had $52.9 \%$ sensitivity and $76 \%$ specificity.

Table (5): The diagnostic validity of total CD24 immunoreactivity.

\begin{tabular}{|c|c|c|c|c|c|}
\hline Diagnosis & Sensitivity & Specificity & PPV & NPV & Accuracy \\
\hline $\begin{array}{l}\text { - Atypical EH Vs. } \\
\text { carcinoma }\end{array}$ & $77.2 \%$ & $76 \%$ & $81.9 \%$ & $70.3 \%$ & $76.7 \%$ \\
\hline $\begin{array}{l}\text { - Atypical EH Vs. } \\
\text { well } \\
\text { differentiated } \\
\text { carcinoma }\end{array}$ & $52.9 \%$ & $76 \%$ & $60 \%$ & $70.3 \%$ & $70 \%$ \\
\hline
\end{tabular}

Association between combined membranous and cytoplasmic CD24 immunostaining and clinicopathological variables of endometrioid carcinoma cases (Table 6):

A statistically significant association was found between combined positive CD24 immunostaining and histological grades $(p=0.035)$. In grade $3,6 / 10$ $(60 \%)$ showed combined positive membranous and cytoplasmic CD24 immunostaining compared to $2 / 15(6.7 \%)$ and $4 / 10(40 \%)$ for grade 1 and 2 , respectively Fig. (5).

Table (6): Association between combined membranous and cytoplasmic CD24 immunostaining and clinicopathological variables of endometrioid carcinoma cases.

\begin{tabular}{|c|c|c|c|c|c|}
\hline \multirow{2}{*}{$\begin{array}{l}\text { Clinicopathological } \\
\text { variables }\end{array}$} & \multirow[b]{2}{*}{ No. } & \multirow{2}{*}{$\begin{array}{c}\text { Negative } \\
\text {-ve/-ve } \\
(\%)\end{array}$} & \multicolumn{2}{|c|}{ Positive } & \multirow{2}{*}{$\begin{array}{c}p- \\
\text { value }\end{array}$} \\
\hline & & & $\begin{array}{l}\text {-ve/+ve or } \\
+ \text { +ve/-ve }(\%)\end{array}$ & $\begin{array}{c}+\mathrm{ve} /+\mathrm{ve} \\
(\%)\end{array}$ & \\
\hline \multicolumn{6}{|l|}{ Age: } \\
\hline$<64$ & 15 & $2(13.3)$ & $6(40)$ & $7(46.7)$ & 0.186 \\
\hline$>64$ & 20 & $8(40)$ & $7(35)$ & $5(25)$ & \\
\hline \multicolumn{6}{|l|}{ Grade: } \\
\hline Grade 1 & 15 & $6(40)$ & $8(53.3)$ & $2(6.7)$ & 0.035 \\
\hline Grade 2 & 10 & $2(20)$ & $4(40)$ & $4(40)$ & \\
\hline Grade 3 & 10 & 0 & $4(40)$ & $6(60)$ & \\
\hline \multicolumn{6}{|l|}{ Myometrial invasion: } \\
\hline$<1 / 2$ & 22 & $7(31.8)$ & $10(45.54)$ & $5(22.7)$ & 0.173 \\
\hline$>1 / 2$ & 13 & $1(7.6)$ & $6 \quad(46.2)$ & $6(46.2)$ & \\
\hline \multicolumn{6}{|l|}{ LN metastasis: } \\
\hline Negative & 17 & $4(23.5)$ & $9(52.9)$ & $4(23.5)$ & 0.063 \\
\hline Positive & 5 & 0 & $1(20)$ & $4(80)$ & \\
\hline \multicolumn{6}{|l|}{ FIGO stage: } \\
\hline Stage I & 29 & $8(27.6)$ & $14(48.3)$ & $7(24.1)$ & 0.183 \\
\hline Stage II-III-IV & 6 & 0 & $2 \quad(33.3)$ & $4(66.7)$ & \\
\hline
\end{tabular}

Test of significance: Chi-square test and f Significant $p$-value $<0.05$ -ve/-ve: -veMEM/-veCYTO. +ve/-ve: +veMEM/-veCYTO.

-ve/+ve: -veMEM/+veCYTO. +ve/+ve: +veMEM/+veCYTO. 
Association between combined positive CD24 immunostaining and LN status was non-significant ( $p=0.063)$, however, combined positive CD24 immunostaining was more frequently seen among cases positive for LN metastasis $4 / 5$ (80\%). Also No association was found between combined pos-

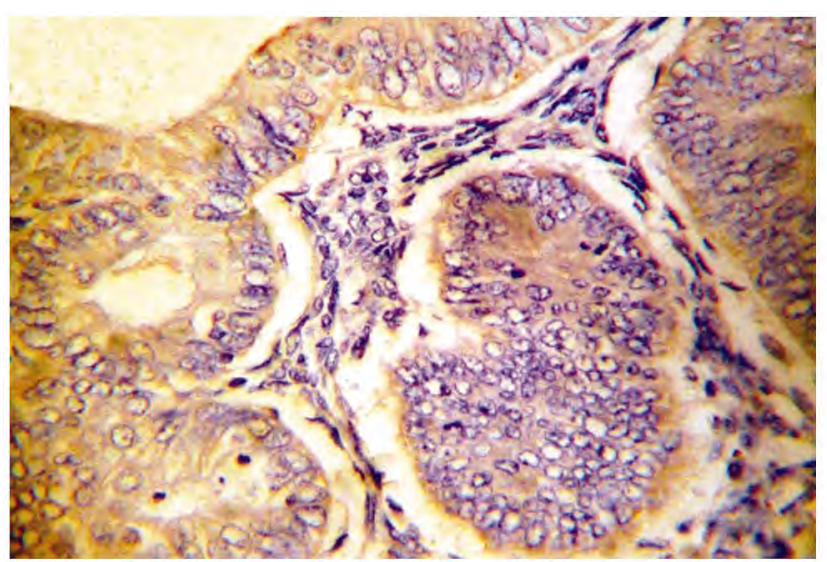

Fig. (4A): Atypical endometrial hyperplasia showing positive memberous CD24 expression (DAB chromogen, haematoxylin counter stain X400).

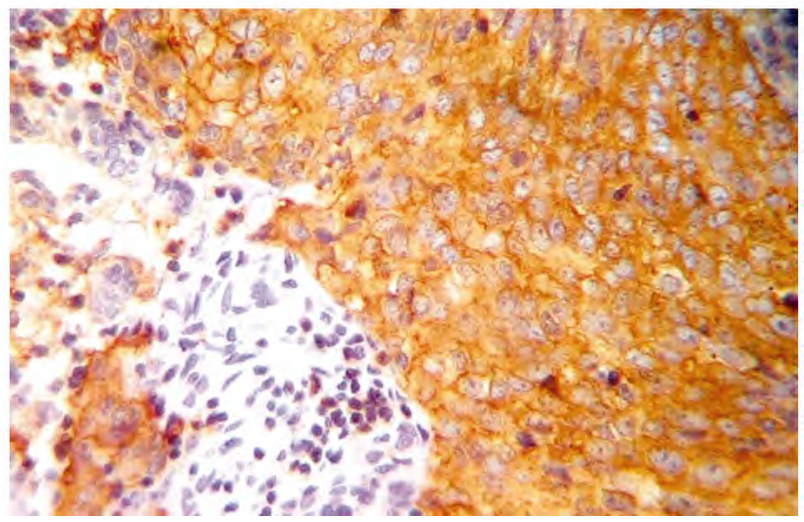

Fig. (5): Poorly differentiated endometrioid carcinoma showing positive membranous and cytoplasmic CD24 expression (DAB chromogen, haematoxylin counter stain X400).

\section{Discussion}

The endometrioid EC are indolent and low stage cancer. It commonly depends on hyperestrogenism and EH [21,22]. Both simple and complex hyperplasia without atypia is not considered preneoplastic forms [23]. Only atypical EH is clearly associated with the subsequent development of adenocarcinoma, so it is important to definitely differentiate between well-differentiated carcinomas and atypical EH [24]

In the present study, well differentiated endometrioid carcinoma representing $42.8 \%$, this was in concordance with previous studies by Ohno et al., itive CD24 immunostaining and FIGO stage ( $p=$ 0.183 ), however, most of advanced stage tumors 4/6 (66.7\%) showed combined positive CD24 immunostaining. For patients' age or myometrial invasion no association were found in combined positive CD24 expression.

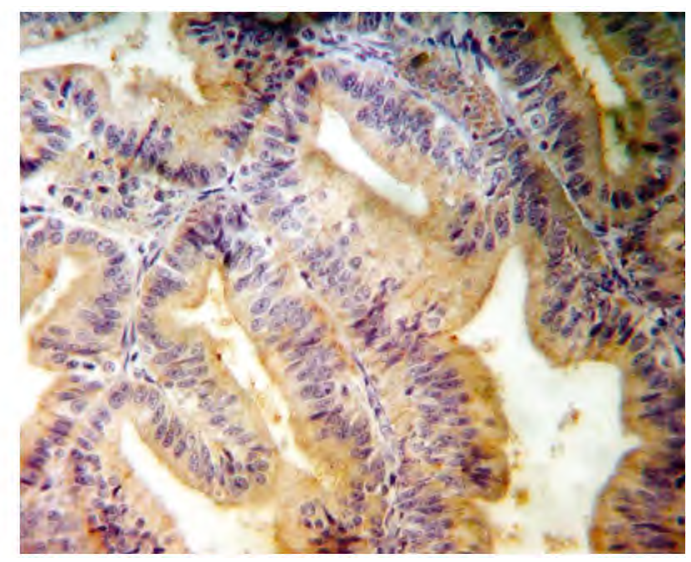

Fig. (4B): Well differentiated endometrioid carcinoma showing positive cytoplasmic CD24 expression (DAB chromogen, haematoxylin counter stain X400).

[25] and Markova et al., [26] who reported well differentiated endometrioid carcinoma ranging from $44.1 \%-48.3 \%$ among their series, while Younes et al., [27] reported that moderately differentiated endometrioid carcinoma represented $58.1 \%$ and in a study done by Abd El-Maqsoud and ElGelany, [28] moderately differentiated endometrioid carcinoma and poorly differentiated endometrioid carcinoma $(37.1 \%)$ was equally represented in their studied cases.

Regarding myometrial invasion, $62.9 \%$ cases showed myometrial invasion $<1 / 2$, while $37.1 \%$ showed myometrial invasion $\geq 1 / 2$, this was in line with Markova et al., [26] andYounes et al., [27] .

For $\mathrm{LN}$ metastasis $77.2 \%$ of cases were negative and $22.8 \%$ were positive for $\mathrm{LN}$ metastasis. This finding in concordance with reported data by Markova et al., [26]. Moreover, much higher frequency of cases negative for nodal metastasis was reported (93.6\% and $92.8 \%$ ) by Ozkara and Corakci, [29] and Ohno et al., [25].

With respect to the stage of endometrioid carcinoma cases in this study, $82.9 \%$ were (stage I) and $17.1 \%$ were advanced stage (II/III/IV stages). These findings were consistent with other studies $[26,27]$ who reported highest occurrence of localized disease ranging from $68.1 \%-81.6 \%$, this confirming that endometrioid carcinoma often produces symptoms at relatively early stages, so this helping in early diagnosis of the disease. 
CD24 protein was first reported to be expressed by pre-B lymphocytes, but it is lost during maturation to plasma cells [5]. CD24 immunoexpression have been reported to be an important marker for diagnosis, tumor progression and cancer-related death in many carcinomas [13-17]

In the current study, CD24 expression was observed in both membrane and cytoplasm of cells. This findings support earlier reports of membrane as well as cytoplasmic localization of CD24 in endometrial tissue [30-32]. As regard expression of membranous CD24 in cyclic endometrium positive membranous expression was detected in $45 \%$ of cases, the proliferative phase and secretory phase cases had $20 \%$ and $70 \%$ positivity, respectively. This was comparable to Kim et al., [31] and this difference was statistically significant as reported by Sundqvist et al., [32]. This up regulation of CD24 membranous expression from proliferative to secretory may suggest that $\mathrm{CD}$ expression has a role in differentiation or maturation of the endometrial glandular cells.

This study showed a statistically significant increase in the expression of membranous CD24 with the progression from $\mathrm{EH}$ without atypia, $13.4 \%$, to atypical EH, $24 \%$, to endometrioid carcinoma, $71.4 \%$. This correlated with the study reported by Kim et al., [31] who reported that membranous CD24 expression significantly enhanced along different lesions; EH without atypia, $13.5 \%$, atypical EH, $33.3 \%$ and endometrioid carcinoma, $64.6 \%$. This finding suggests that CD24 expression may be linked with progression from precancerous lesion to carcinomatous lesion. A similar was documented in previous studies on colon cancer [33], and ovary, as Aktas et al., [34] and Moulla et al., [35] detected that the CD24 staining was significantly increased in ovarian carcinomas and borderline tumors compared to normal ovaries and cystadenomas. Also, this change of membranous CD24 expression in hyperplasia and carcinoma, in which there is lowering of expression in the hyperplastic lesions, followed by a remarkable increasing in endometrioid carcinoma, suggesting that membranous CD24 expression may be used as a diagnostic tool for the differential diagnosis between endometrioid carcinoma and atypical EH, which is a common diagnostic challenge, especially in the D \& C Biopses.

In the current study, the positive expression rates of membranous CD24 were significantly lowered in either EH without atypia or atypical $\mathrm{EH}$ lesions than those of the cyclic endometrium. Moreover, no significant difference was found between $\mathrm{EH}$ without atypia and atypical $\mathrm{EH}$ cases. These findings were consistent with Kim et al., [31]

On studying the association of membranous CD24 expression and different clinicopathological variables in endometrioid carcinoma cases, this study showed that positive significant association between membranous CD24 immunostaining and histological grades. This was in accordance with Karahan et al., [30] and Kim et al., [31].

Regarding CD24 immunostaining and myometrial invasion and FIGO stage as well, the present study demonstrates a significant positive association between them implying the role of CD24 in cancer progression. On contrary, Karahan et al., [30], and Kim et al., [31] did not found such association. These differences are related to the use of different scoring method and different antibodies.

Concerning LN status in the studied cases, no significant association was found between membranous CD24 immunostaining and LN status, although, all cases positive for LN metastasis were positive for membranous CD24 immunostaining versus $70.6 \%$ positivity for cases negative for LN metastasis. This was in accordance to Karahan et al., [30] and Kim et al., [31] who reported no significant association between membranous CD24 expression and LN metastasis. Similarly this finding reported in breast carcinoma [13] and ovarian carcinoma [36]

On studying cytoplasmic CD24 immunoexpression in cyclic endometrium; positive CD24 expression was detected in $35 \%$ of cases. These findings were in line with Kim et al., [31] who reported expression in $44.4 \%$ in their series.

No significant difference was detected between the expression of cytoplasmic CD24 and normal cyclic endometrium. This was comparable with Kim et al., [31] who found $41.7 \%$ and $47.8 \%$ positivity for proliferative phase and secretory phase cases, respectively.

A significant increase in cytoplasmic CD24 expression with lesion progression was detected in this study where the expression rates were $6.7 \%$, $20 \%, 40 \%$ for $\mathrm{EH}$ without atypia, atypical $\mathrm{EH}$ and endometrioid carcinoma, respectively. Such finding demonstrated also by Kim et al., [31] where cytoplasmic CD24 expression was seen in 5.8\%, 29.2\%, $39 \%$ for $\mathrm{EH}$ without atypia, atypical $\mathrm{EH}$ and endometrioid carcinoma, respectively. Karahan et al., [30] reported lower rate of expression (28.9\%) in endometrioid carcinoma cases. This different 
rate of expression may be attributed to different clone used by that study ( $\mathrm{Ab} 2$, clone $24 \mathrm{C02}$ ).

Concerning cytoplasmic CD24, in this study, the positive rates of cytoplasmic CD24 immunoexpression in various types of hyperplastic lesions were also lower than those of the cyclic endometrium and endometrioid carcinoma. There was a significant increase in the expression of cytoplasmic $\mathrm{CD} 24$ in EH without atypia cases as compared to its expression in cyclic endometrium and endometrioid carcinoma cases. But no significant difference was found between cyclic endometrium and atypical EH cases or cyclic endometrium and endometrioid carcinoma cases as well. This was in line with findings of Kim et al., [31]

Similarly, no significant differences were found between atypical EH cases and EH without atypia cases or endometrioid carcinoma cases regarding cytoplasmic CD24 expression. This was in concordance with Kim et al., [31].

Among cases of endometrial carcinoma this study demonstrates a positive significant association between tumor grade and cytoplasmic localization of CD24. However no significant associations were detected with other clinicopathological variables. Similar findings were reported by Karahan et al., [30] and Kim et al., [31]

In the current study, total positive CD24 expression was detected in $77.1 \%$ of endometrioid carcinoma. This correlated with the study performed by Karahan et al., [30] who reported total positive CD24 expression in $77.3 \%$ of their endometrioid carcinoma cases.

Based on previous reports and in the light of our findings, the intracellular localization of CD24 in cancer cells has been reported to express different biological features of cancer behavior. Membranous or cytoplasmic CD24 overexpression was closely related to adverse factors. However, the significance of CD24 overexpression was not consistent among carcinomas arising in different organs [15]. As in breast carcinoma, Kristiansen et al., [20] and Athanassiadou et al., [13] found no association between membranous CD24 immunoreactivity and clinicopathological parameters. While Bircan et al., [37] reported that membranous immunostaining was significantly positively correlated with tumor grade and there was no such an association with the cytoplasmic staining. In ovarian carcinoma, Kristiansen et al., [38] and Surowiak et al., [36] found no association for either membranous or cytoplasmic CD24 immunoreactivity with any of clinicopathological variables. But Moulla et al., [35], reported that high-grade carcinomas and carcinomas with metastases to the omentum had considerably higher CD24 expression.

Accumulative data from this study and those of the literature $[30,31]$ detected the positive correlation between adverse factors and membranous CD24 expression and the positive relationship with lesion progression for membranous CD24 immunoexpression and combined positive CD24 immunostaining as well. This supports the view that membranous CD24 expression and combined positive CD24 immunostaining as well may suggest a more aggressive phenotype in endometrioid carcinoma. In addition, the significant difference in membranous CD24 expression between endometrioid carcinoma and atypical $\mathrm{EH}$ is useful for the differential diagnosis between endometrioid carcinoma and atypical EH.

To the best of our knowledge, this is the first study that evaluated the sensitivity and specificity of CD24 expression in differentiating atypical EH from endometrioid carcinoma and atypical $\mathrm{EH}$ from well-differentiated endometrioid carcinoma as well. We found that membranous CD24 expression had more sensitivity and specificity than cytoplasmic CD24 expression which was not sensitive but was specific for such distinction. However, total positive CD24 expression is more sensitive $(77.2 \%)$ than positive membranous or cytoplasmic expression alone $(71.4 \%$ and $40 \%$, respectively) in distinction of endometrioid carcinoma from atypical EH. Furthermore, total positive CD24 expression is more sensitive (52.9\%) than membranous or cytoplasmic expression alone (46.7\% and $13.3 \%$ ) in distinction of well differentiated endometrioid carcinoma from atypical EH.

This study demonstrates that CD24 is expressed in normal cyclic endometrium, hyperplastic lesion and endometrial carcinoma. The up regulation of membernous CD24 expression from proliferative to secretory endometrium indicates that CD24 has a role in differentiation of the endometrial glands. Enhanced membernous CD24 expression can be used as a diagnostic marker in distinction of well differentiated endometrioid carcinoma from atypical EH however total CD24 (membrances and cytoplasmic) expression is more sensitive in this Challenge. CD24 overexpression is involved in progression of endometrial carcinoma.

\section{References}

1- FERLAY J., SOERJOMATARAM I., DIKSHIT R., ESER S., MATHERS C., REBELO M., PARKIN D.M., FORMAN D. and BRAY F.: Cancer incidence and mortality 
worldwide: Sources, methods and major patterns in GLOBOCAN 2012. Int. J. Cancer, 136: E359-86, 2015.

2- JEMAL A., BRAY F., CENTER M.M., FERLAY J., WARD E. and FORMAN D.: Global cancer statistics. CA Cancer J. Clin., 61: 69-90, 2011.

3- MOKHTAR N., ADEL I. and GOUDA I.: Malignant Female Genital System Tumors. In: Cancer Pathology Registry 2003-2004 and Time Trend Analysis: Chapter 8. 1 st Ed. National Cancer Institute and Sanofi Aventis, Egypt, 66-74, 2007.

4- IBRAHIM A.S., KHALED H.M., MIKHAIL N.N., BARAKA H. and KAMEL H.: Cancer incidence in Egypt: Results of the national population-based cancer registry program. J. Cancer Epidemiol., 437971, 2014.

5- CHAPPEL M.S., HOUGH M.R., MITTEL A., TAKEI F., KAY R. and HUMPHRIES R.K.: Cross-linking the murine heat-stable antigen induces apoptosis in B cell precursors and suppresses the anti-CD40-induced proliferation of mature resting B lymphocytes. J. Exp. Med., 184: 163949, 1996.

6- KAY R., ROS TEN P.M. and HUMPHRIES R.K.: CD24, a signal transducer modulating B cell activation responses, is a very short peptide with a glycosyl phosphatidylinositol membrane anchor. J. Immunol., 147: 1412-6, 1991.

7- LI O., ZHENG P. and LIU Y.: CD24 expression on T cells is required for optimal $\mathrm{T}$ cell proliferation in lymphopenic host. J. Exp. Med., 200: 1083-9, 2004.

8- ISRAEL E., KAPELUSHNIK J., YERMIAHU T., LEVI I., YANIV I., SHPILBERG O. and SHUBINSKY G.: Expression of CD24 on CD19-CD79a+ early B-cell progenitors in human bone marrow. Cell Immunol, 236: 1718, 2005.

9- ELGHETANY M.T. and PATEL J.: Assessment of CD24 expression on bone marrow neutrophilic granulocytes: CD24 is a marker for the myelocytic stage of development. Am. J. Hematol., 71: 348-9, 2002.

10- BATES M.E., LIU L.Y., ESNAULT S., STOUT B.A., FONKEM E., KUNG V., SEDGWICK J.B., KELLY E.A., et al.: Expression of interleukin-5-and granulocyte macrophage-colony-stimulating factor-responsive genes in blood and airway eosinophils. Am. J. Respir. Cell. Mol. Biol., 30: 736-43, 2004.

11- De HEUSCH M., GARZE V., MALISZEWSKI C., URBAIN J., LIU Y. and MOSER M.: The heat stable antigen (CD24) is not required for the generation of CD4+ effector and memory $\mathrm{T}$ cells by dendritic cells in vivo. Immunol. Lett, 94: 229-37, 2004.

12- SAGIV E. and ARBER N.: The novel oncogene CD24 and its arising role in the carcinogenesis of the GI tract: From research to therapy. Expert Rev. Gastroenterol. Hepatol., 2: 125-33, 2008.

13- ATHANASSIADOU P., GRAPSA D., GONIDI M., ATHANASSIADOU A.M., TSIPIS A. and PATSOURIS E.: CD24 expression has a prognostic impact in breast carcinoma. Pathol. Res. Pract., 205: 524-33, 2009.

14- BAUMHOER D., RIENER M.O., ZLOBEC I., TORNILLO L., VOGETSEDER A., KRISTIANSEN G., DIETMAIER W., HARTMANN A., WUENSCH P.H., SESSA F., RUEMMELE P. and TERRACCIANO L.M.: Expres- sion of CD24, P-cadherin and S100A4 in tumors of the ampulla of Vater. Mod. Pathol., 22: 306-13, 2006.

15- LEE H.J., CHOE G., JHEON S., SUNG S.W., LEE C.T. and CHUNG J.H.: CD24, a novel cancer biomarker, predicting disease-free survival of non-small cell lung carcinomas: A retrospective study of prognostic factor analysis from the viewpoint of forthcoming (seventh) new TNM classification. J. Thorac. Oncol., 5: 649-57, 2010.

16- WANG W., WANG X., PENG L., DENG Q., LIANG Y., QING H. and JIANG B.: CD24-dependent MAPK pathway activation is required for colorectal cancer cell proliferation. Cancer Sci., 101: 112-9, 2010.

17- OVERDEVEST J.B., THOMAS S., KRISTIANSEN G., HANSEL D.E., SMITH S.C. and THEODORESCU D.: CD24 offers a therapeutic target for control of bladder cancer metastasis based on a requirement for lung colonization. Cancer Res., 71: 3802-11, 2011.

18- SILVERBERG S.G., KURMAN R.J., NOGALES F., MUTTER G.L., KUBIK-HUCH R.A. and TAVASSOLI F.A.: Tumors of the uterine corpus. In: Tavassoli FA, Devilee P, editors. Pathology and genetics of tumours of the breast and female genital organs. World Health Organization classification of tumours. Lyon, France: IARC Press, 217-32, 2015.

19- PECORELLI S.: Revised FIGO staging for carcinoma of the vulva, cervix, and endometrium. Int. J. Gynaecol. Obstet., 105: 103-4, 2009.

20- KRISTIANSEN G., WINZER K.J., MAYORDOMO E., BELLACH J., SCHLUNS K., DENKERT C., DAHL E., PILARSKY C., ALTEVOGT P., GUSKI H. and DIETEL M.: CD24 expression is a new prognostic marker in breast cancer. Clin. Cancer Res., 9: 4906-13, 2003.

21- DOLL A., ABAL M., RIGAU M., MONGE M., GONZALEZ M., DEMAJO S., COLAS E., LLAURADO M., ALAZZOUZI H., PLANAGUMA J., LOHMANN M.A., GARCIA J., CASTELLVI S., RAMON Y. CAJAL J., GIL-MORENO A., XERCAVINS J., ALAMEDA F. and REVENTOS J.: Novel molecular profiles of endometrial cancer-new light through old windows. J. Steroid. Biochem. Mol. Biol., 108: 221-9, 2008.

22- LLOBET D., PALLARES J., YERAMIAN A., SANTACANA M., ERITJA N., VELASCO A., DOLCET X. and MATIAS-GUIU X.: Molecular pathology of endometrial carcinoma: Practical aspects from the diagnostic and therapeutic viewpoints. J. Clin. Pathol., 62: 777-85, 2009.

23- PRAT J., GALLARDO A., CUATRECASAS M. and CATASUS L.: Endometrial carcinoma: Pathology and genetics. Pathology, 39: 72-87, 2007.

24- DAGLI A.F., AYDIN S., KOCDOR H., GURATES B., SAHIN I., CATAK Z., OZERCAN M.R. and OZERCAN I.H.: Ghrelin expression of endometrium hyperplasia and endometrioid carcinoma. Gynecol. Endocrinol., 27: 199204, 2011.

25- OHNO S., DOHI S., OHNO Y., KYO S., SUGIYAMA H., SUZUKI N. and INOUE M.: Immunohistochemical detection of WT1 protein in endometrial cancer. Anticancer. Res., 29: 1691-5, 2009.

26- MARKOVA I., DUSKOVA M., LUBUSKY M., KUDELA M., ZAPLETALOVA J., PROCHAZKA M. and PILKA R.: Selected immunohistochemical prognostic factors in 
endometrial cancer. Int. J. Gynecol. Cancer, 20: 576-82, 2010.

27- YOUNES S.F., AIAD H., KANDIL M. and EL-KALASHY F.S.: Expression and Clinical Significance of Ghrelin in Endometrial Hyperplasia and Carcinoma of Egyptian Patients. Ultrastructural Pathology, 39 (3): 207-13, 2015.

28- ABD EL-MAQSOUD N.M. and EL-GELANY S.: Differential Expression Patterns of PTEN in Cyclic, Hyperplastic and Malignant Endometrium: Its Relation with ER, PR and Clinicopathological Parameters. J. Egypt Natl. Canc. Inst., 21: 323-31, 2009.

29- OZKARA S.K. and CORAKCI A.: Significantly decreased P27 expression in endometrial carcinoma compared to complex hyperplasia with atypia (correlation with p53 expression). Pathol. Oncol. Res., 10: 89-97, 2004.

30- KARAHAN N., GUNEY M., ORAL B., KAPUCUOGLU N. and MUNGAN T.: CD24 expression is a poor prognostic marker in endometrial carcinoma. Eur. J. Gynaecol. Oncol., 27: 500-4, 2006.

31- KIM K.H., CHOI J.S., KIM J.M., CHOI Y.L., SHIN Y.K., LEE H.C., SEONG I.O., KIM B.K., CHAE S.W. and KIM S.H.: Enhanced CD24 expression in endometrial carcinoma and its expression pattern in normal and hyperplastic endometrium. Histol. Histopathol., 24: 309-16, 2009.

32- SUNDQVIST J., ANDERSSON K.L., SCARSELLI G., GEMZELL-DANIELS SON K. and LALITKUMAR P.G.: Expression of adhesion, attachment and invasion markers in eutopic and ectopic endometrium: A link to the aetiology of endometriosis. Hum. Reprod., 27: 2737-46, 2012.
33- CHOI Y., XUAN Y., LEE S., PARK S., KIM W., KIM H and KIM S.: Enhanced CD24 Expression in Colorectal Cancer Correlates with Prognostic Factors. The Korean Journal of Pathology, 40: 103-11, 2006.

34- AKTAS I.Y., BUGDAYCI M. and USUBUTUN A.: Expression of p16, p53, CD24, EpCAM and calretinin in serous borderline tumors of the ovary. Turk. Patoloji. Derg., 28: 220-30, 2012.

35- MOULLA A., MILIARAS D., SIOGA A., KAIDOGLOU A. and ECONOMOU L.: The immunohistochemical expression of CD24 and CD171 adhesion molecules in borderline ovarian tumors. Pol. J. Pathol., 64: 180-4, 2013.

36- SUROWIAK P., MATERNA V., KAPLENKO I., SPACZYNSKI M., DIETEL M., KRISTIANSEN G., LAGE H. and ZABEL M.: Unfavorable prognostic value of CD24 expression in sections from primary and relapsed ovarian cancer tissue. Int. J. Gynecol. Cancer, 16: 515-21, 2006.

37- BIRCAN S., KAPUCUOGLU N., BASPINAR S., INAN G. and CANDIR O.: CD24 expression in ductal carcinoma in situ and invasive ductal carcinoma of breast: An immunohistochemistry-based pilot study. Pathol. Res. Pract., 202: 569-76, 2006.

38- KRISTIANSEN G., DENKERT C., SCHLUNS K., DAHL E., PILARSKY C. and HAUPTMANN S.: CD24 is expressed in ovarian cancer and is a new independent prognostic marker of patient survival. Am. J. Pathol., 161: 1215-21, 2002. 


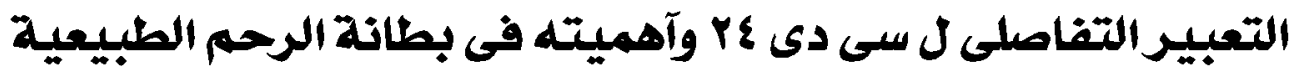

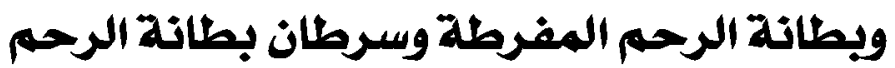

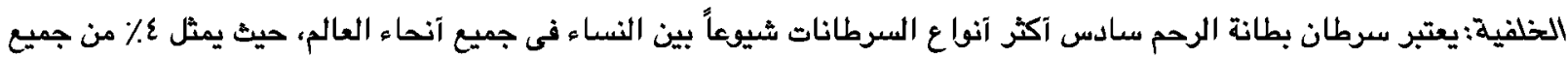

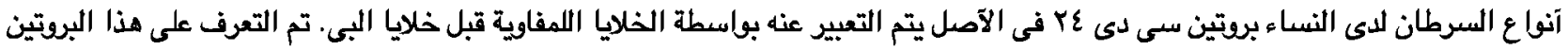

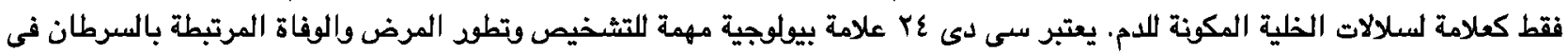

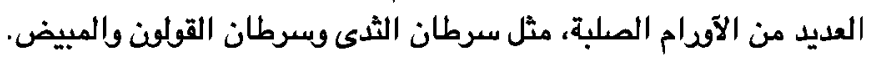

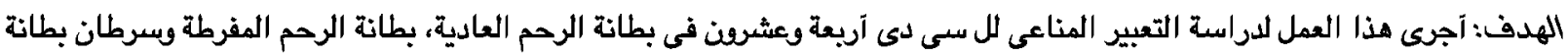

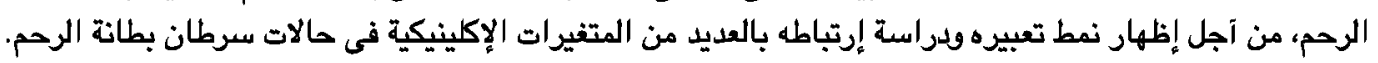

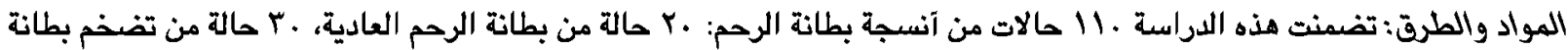

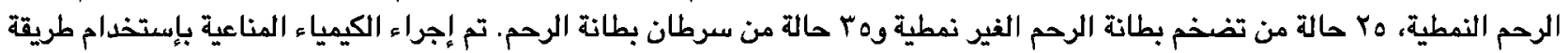
الآقيدين البيوتين بيركسيديز.

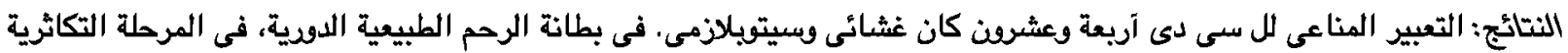

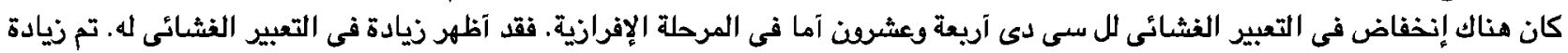

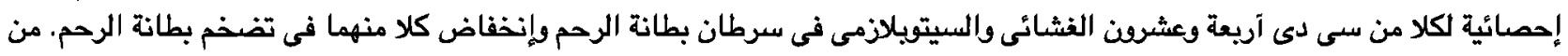

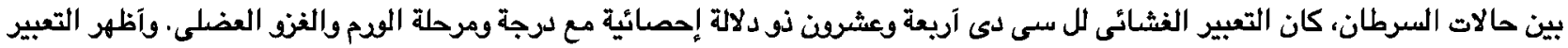

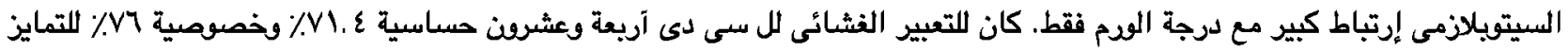

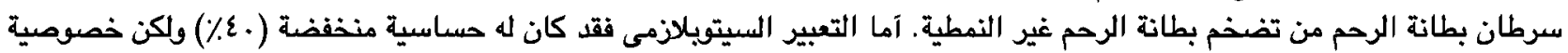

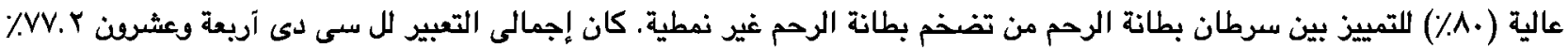

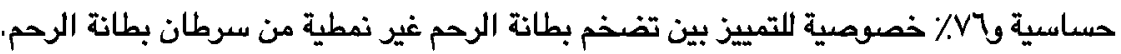

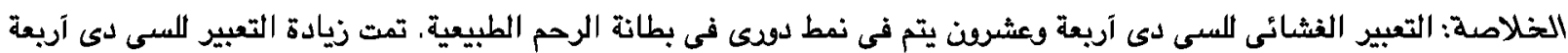

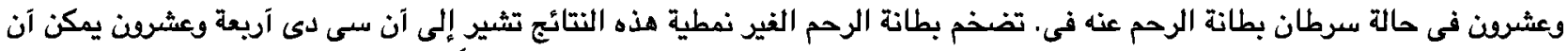

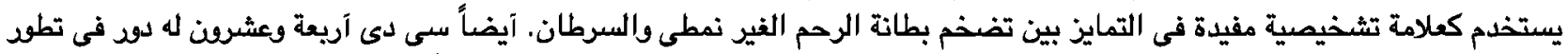

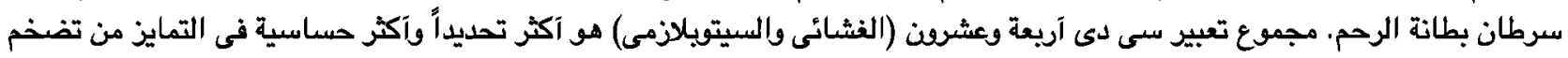
بطانة الرحم غير نمطية من سرطان بطانة الرحم. 